\title{
IV ENCONTRO DE GRUPOS PET CEFET-MG E I ENCONTRO REGIONAL DE GRUPOS PET: IV INTERPET A I ERPET
}

Ludmila A. Oliveira- ludmila.nepomuceno.cefetmg@ gmail.com

Avenida Monsenhor Luiz de Gonzaga, 103

37250-000 - Nepomuceno - MG

Letícia S. Santos - leticiasotnas.ls@gmail.com

Centro Federal de Educação Tecnológica de Minas Gerais, Departamento de Elétrica

Avenida Monsenhor Luiz de Gonzaga, 103

37250-000 - Nepomuceno - MG

Reginaldo B. Fernandes- reginaldo@ cefetmg.br

Avenida Monsenhor Luiz de Gonzaga, 103

37250-000 - Nepomuceno - MG

Samuel S. F. Terra - samueldesouzaferreiraterra@ gmail.com

Avenida Monsenhor Luiz de Gonzaga, 103

37250-000 - Nepomuceno - MG

Sara L.Silva - sara.luiiza13@gmail.com

Avenida Monsenhor Luiz de Gonzaga, 103

37250-000 - Nepomuceno - MG

Resumo:Este artigo apresenta as atividades desenvolvidas durante o IV Encontro de Grupos PET do CEFET-MG (InterPET) e I Encontro Regional de grupos PET(ERPET), que ocorreram simultaneamente no período entre 29 e 31 de agosto de 2019, no CEFET-MG Campus Nepomuceno. O InterPET é um evento realizado desde 2016 nos Campus do CEFET-MG, com o intuito de discutir e propor mudanças para melhoria dos grupos PET do CEFET-MG, bem como, relatar as atividades desenvolvidas por cada grupo. O I ERPET foi um evento idealizado pelo grupo PET Engenharia Elétrica do CEFET-MG Campus Nepomuceno que teve como objetivo debater as vertentes do Programa de Educação Tutorial com os grupos PET da região, assim como, expor os trabalhos desenvolvidos por cada grupo. Além da comunidade petiana, o evento foi aberto a comunidade externa. Durante a programação do ERPET, diversas atividades foram destinadas a alunos de escolas Municipais e Estaduais da cidade de Nepomuceno. Com a realização dos eventos foi possível elucidar a importância da tríade ensino, pesquisa e extensão, tanto para comunidade acadêmica quanto para a comunidade externa, contribuindo para uma reflexão sobre a função social dos grupos PET como agentes transformadores fora e dentro da universidade.

Palavras-chave:PET. Extensão. Ensino. Educação. 


\section{INTRODUÇÃO}

É responsabilidade das universidades desenvolver ações que promovam a consolidação da indissociabilidade entre Ensino, Pesquisa e Extensão, em conformidade com a Constituição Federal de 1988 que dispõe do artigo 207, que cita "As universidades gozam de autonomia didático-científica, administrativa e de gestão financeira e patrimonial, e obedecerão ao princípio de indissociabilidade entre ensino, pesquisa e extensão" (Brasil, 1996).

Nesse contexto, o programa de educação tutorial (PET) se torna primordial para impulsionar o princípio da indissociabilidade nas universidades. Com o objetivo de desenvolver ações de ensino, pesquisa e extensão o programa visa promover uma formação acadêmica ampla e de qualidade para os discentes envolvidos diretamente ou indiretamente com o programa, assim como, estimular valores que endossam a formação de cidadãos com responsabilidade e consciência social. Diante disso, os grupos PET representam uma alternativa para realização de eventos acadêmicos com intuito de divulgar as atividades fundamentadas na tríade ensino, pesquisa e extensão, com participação da comunidade. Eventos técnicocientíficos como palestras, minicursos, congressos, seminários, assumem um papel de grande importância para divulgação do saber em que, a troca de experiências e conhecimentos promovem a integração do ensino e a expansão cultural e científica na sociedade, possibilitando o enriquecimento do saber acadêmico. De acordo com a Secretaria de Educação Superior (SESU) o contato sistemático tanto com a comunidade acadêmica como um todo quanto com a comunidade externa, promove a troca de experiências e conhecimentos em um processo de mútua aprendizagem.

No que se refere a relação universidade e comunidade, Nunes e Silva (2012) ponderam:

O fortalecimento da relação universidade/sociedade prioriza a superação das condições de desigualdades e exclusão existentes. Através de projetos sociais, a universidade socializa seu conhecimento e disponibiliza seus serviços, exercendo sua responsabilidade social, ou mesmo sua missão: o compromisso com a melhoria da qualidade de vida dos cidadãos (NUNES; SILVA, 2012, p. 121).

Diante desse exposto, o grupo PET de Engenharia Elétrica (PETEE) do CEFET-MG Campus Nepomuceno busca viabilizar ações que levem conhecimento e assistência tanto para a comunidade acadêmica quanto para o município de Nepomuceno. Fundado em outubro de 2017, o grupo é constituído por 8 alunos bolsistas e 2 alunos voluntários que estão sob tutela de 1 tutor e 3 cotutores. O PETEE atua com a temática de eficiência enérgica e tem como objetivo proporcionar aos discentes do curso de Engenharia Elétrica uma gama nova e diversificada de conhecimento técnico e acadêmico, assim como, contribuir para formação de profissionais éticos e com responsabilidade social.

O presente trabalho visa relatar como foi a organização e realização do IV Encontro de Grupos PET do CEFET-MG (INTERPET) e do I Encontro Regional de Grupos PET (ERPET), mostrando como foi desenvolvido cada evento, incentivando a realização de outras iniciativas que promovam a democratização, divulgação e popularização da ciência.

\section{IV INTERPET}

O InterPET é realizado desde 2016 nos Campus do Centro Federal de Educação Tecnológica de Minas Gerais, cujos objetivos são: a troca de experiências, reflexões, e discussões acerca do desenvolvimento do Programa, em especial no que se refere a articulação 
entre ensino, pesquisa e extensão, bem como a apresentação de pesquisas e trabalhos desenvolvidos pelos grupos.

Além disso o evento tem também o intuito de discutir e propor mudanças que possam auxiliar no aperfeiçoamento dos grupos PET do CEFET-MG, contribuindo para o intercâmbio de conhecimento entre os grupos, além de fomentar a formação acadêmica dos petianos. Durante a quarta edição do InterPET, realizado pelo PETEE no Campus Nepomuceno, ocorreram várias atividades como a apresentação de artigos e atividades executadas por cada grupo PET durante o ano além de palestras e minicursos.

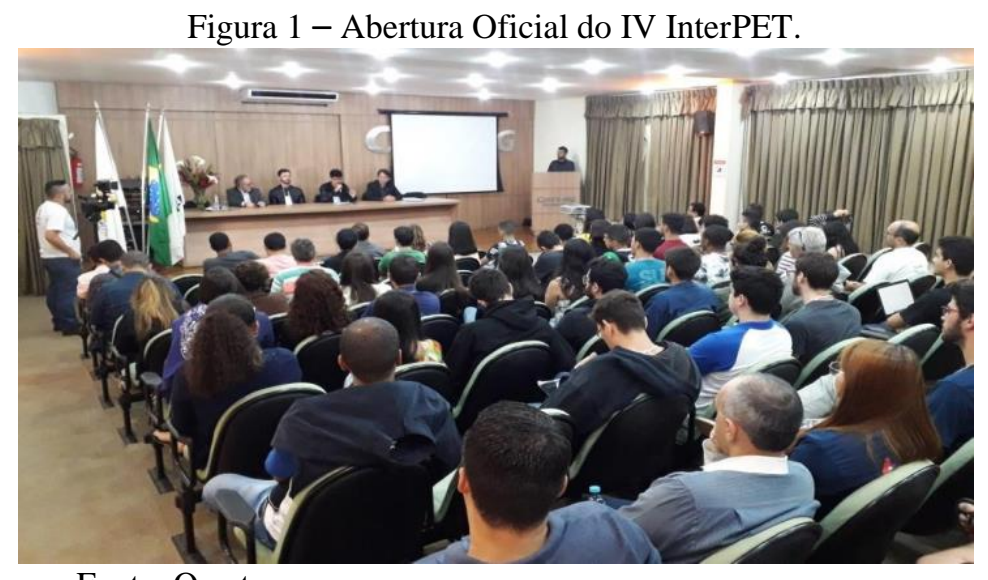

Fonte: $\mathrm{O}$ autor.

\subsection{Atividades dos Grupos PET}

Com o objetivo de promover a troca de experiências entre os grupos e expor as atividades desenvolvidas por cada um durante o ano, há sempre um espaço reservado para a apresentação dos Grupos PET.

$\mathrm{Na}$ edição de 2019 todos os 10 grupos PET estiveram presentes no InterPET do Centro Federal de Educação Tecnológica de Minas Gerais, além do PET Engenharia Elétrica, sendo eles de 6 Campus distintos do CEFET-MG. A Tabela 1 apresenta os grupos participantes e seus respectivos Campus.

Tabela 1: Grupos PET CEFET-MG

\begin{tabular}{c|c}
\hline Grupo PET & Campus \\
\hline $\begin{array}{c}\text { COMPET - PET Engenharia de } \\
\text { Computação }\end{array}$ & Belo Horizonte \\
Filosofia & Belo Horizonte \\
\hline ConecTTE - PET Ciências Sociais e & Belo Horizonte \\
\hline PET ADM - PET Administração & Belo Horizonte \\
\hline $\begin{array}{c}\text { PET Ambiental } \\
\text { Ambient PET Engenharia }\end{array}$ & Curvelo \\
\hline PET ENCAUT - PET Engenharia Civil & Leopoldina \\
Controle e Automação & Araxá \\
\hline PET Engenharia de Automação Industrial & Belo Horizonte \\
\hline PET Materiais - PET Engenharia de \\
Materiais
\end{tabular}


(C) COBENCE

"Os desafios para formar hoje o engenheiro do amanhã"
$\mathrm{Ol}$ a $\mathrm{O3}$ de dezembro Evento On-line

\begin{tabular}{c|c}
\hline $\begin{array}{c}\text { PET Mecatrônica - PET Engenharia } \\
\text { Mecatrônica }\end{array}$ & Divinópolis \\
\hline PET Minas - PET Engenharia de Minas & Araxá \\
\hline PETEE - PET Engenharia Elétrica & Nepomuceno \\
\hline
\end{tabular}

Fonte: Adaptada CEFET-MG

As apresentações dos grupos foram essenciais para que todos tivessem pleno conhecimento de quais são as vertentes trabalhadas e projetos desenvolvidos por cada um deles, podendo inclusive auxiliar na elaboração de novas atividades que possam agregar a sociedade.

\subsection{Resumos Expandidos}

Além da apresentação geral das atividades desenvolvidas pelos grupos, o evento contou também com a apresentação de trabalhos específicos dos mesmos. Durante o processo de inscrição dos petianos os grupos puderam submeter até 3 resumos expandidos cujo conteúdo fosse de projetos específicos desenvolvidos por eles sendo que era necessária a submissão de pelo menos um resumo por grupo PET.

Após a avaliação dos resumos todos os grupos tiveram pelo menos 1 trabalho selecionado. A apresentação foi realizada de forma oral sendo possível a utilização de dispositivos de simulação, vídeos e amostra de protótipos.

Figura 2-Apresentação de Resumos.
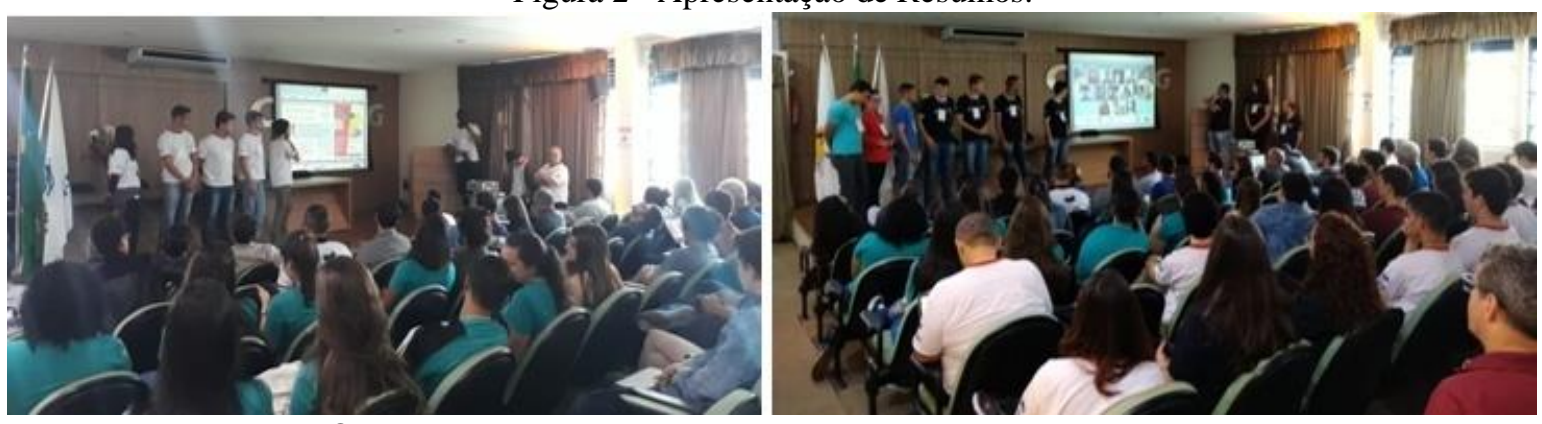

Fonte: $\mathrm{O}$ autor.

A partir da seleção dos trabalhos foi gerado um caderno de resumos do IV InterPET, organizado por Joana Ancila Pessoa Forte Avelino e Ludmila Aparecida de Oliveira. Ao todo foram 17 resumos expandidos dos diferentes grupos PET presentes no CEFET-MG. A Figura 4 mostra a capa do caderno de resumos elaborado pelas organizadoras. 
(C) COBENCE 2020

"Os desafios para formar hoje o engenheiro do amanhã"
$\mathrm{O1} \mathrm{a} \mathrm{O3}$ de dezembro Evento On-line

Figura 3 - Capa do caderno de resumos.

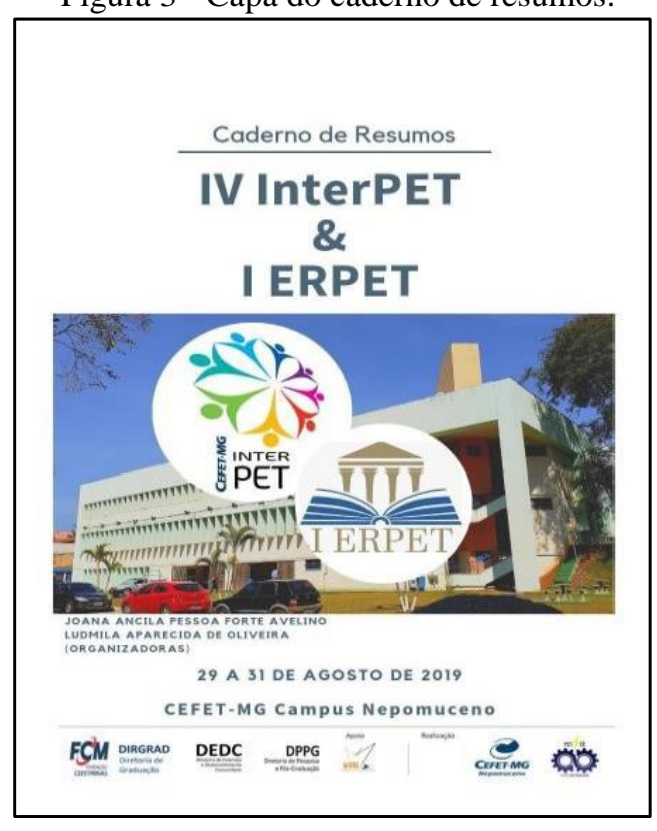

Fonte: $\mathrm{O}$ autor.

\subsection{Palestras e Minicursos}

Com o objetivo de capacitar e auxiliar os petianos na vida acadêmica e profissional foram ofertadas também palestras e minicursos de diversas áreas do conhecimento como administração, engenharias e também outros assuntos relevantes.

Ao todo foram oferecidos 4 (quatro) minicursos e 2 (duas) palestras, foram eles: Palestras:

- O programa Futura-se: Reflexão crítica a respeito do futuro dos projetos de Pesquisa e Extensão voltados para o desenvolvimento de tecnologias sociais e o compromisso com demandas que não estejam restritas apenas aos interesses privados do mercado.

- Como começar a investir: Noções básicas de como iniciar o seu próprio negócio.

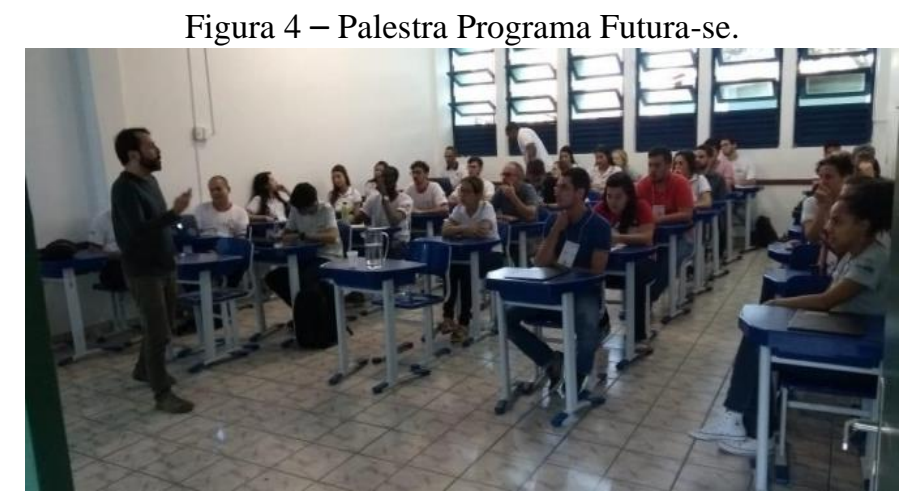

Fonte: $\mathrm{O}$ autor.

Minicursos:

- Identificação Mineral - Curso básico sobre como identificar e diferenciar determinados minerais e rochas. 
- Jogo Bolsa Mineral - Aplicação do jogo Bolsa Mineral, que consiste em uma modificação do jogo Banco Imobiliário para a mineração.

- Oficina Canvas - Modelo de Negócios: Oficina cujo intuito foi ensinar os participantes a criar o seu próprio modelo de negócio canvas, fazendo com que se perceba a viabilidade do empreendimento.

- Programação do Robô Móvel do Projeto Sementes - O objetivo do minicurso foi proporcionar aos interessados em robótica móvel uma introdução conceitual, didática e prática.

Figura 5 -Minicurso Identificação Mineral.

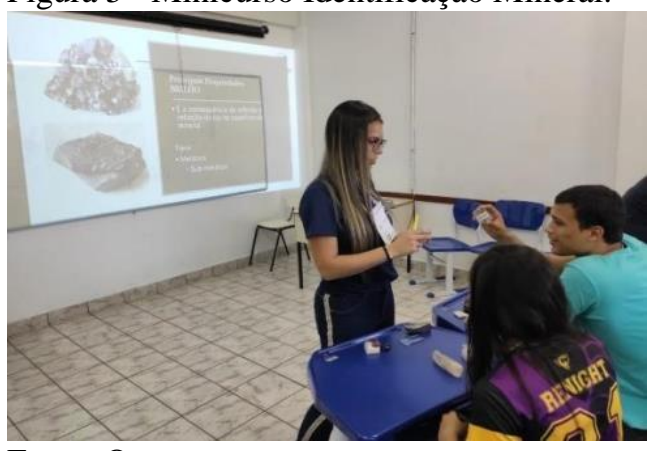

Fonte: $\mathrm{O}$ autor.
Figura 6-Minicurso Bolsa Mineral.

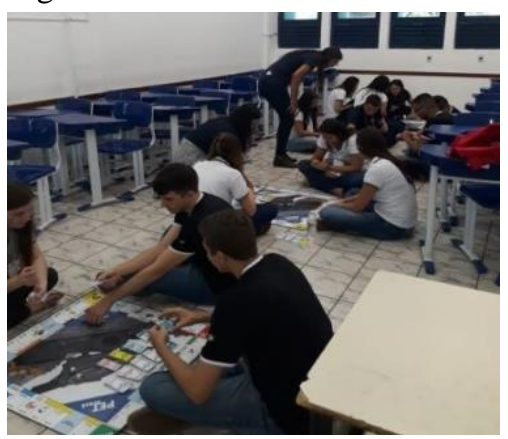

Fonte: $\mathrm{O}$ autor.

\section{I ERPET}

A primeira edição do Encontro Regional de Grupos PETs - ERPET foi realizada em 2019 no Centro Federal de Educação Tecnológica de Minas Gerais, CEFET-MG Campus Nepomuceno. O evento foi uma iniciativa do Grupo PET Engenharia Elétrica, tendo como objetivo apresentar o Programa de Educação Tutorial à comunidade, bem como a realização da troca de experiências entre grupos PETs da região.

O I ERPET contou com a participação de escolas públicas da região, para os quais foram elaborados palestras, minicurso e uma mesa redonda, com temas relacionados ao uso consciente de energia elétrica. Grupos PETs da Universidade Federal de Lavras - UFLA também participaram do evento através da apresentação de atividades desenvolvidas e experiências vivenciadas pelos grupos ao longo do programa.

\subsection{Palestras}

Durante o evento foram ofertadas diversas palestras com o intuito de apresentar o Programa de Educação Tutorial, que desenvolve atividades acadêmicas no âmbito de pesquisa, ensino e extensão, à comunidade bem como evidenciar questões relacionadas ao uso consciente de energia. No total foram realizadas 3 palestras:

- PET - Programa de Educação Tutorial: O Que, Quem, Como e Para Que? Apresentação do programa a toda comunidade, ministrada pelos petianos Ludmila Aparecida de Oliveira e Ualifer Abreu da Silva;

- Eficiência Energética: Apresentar a importância de fontes mais eficientes de energia elétrica, direcionada à alunos do ensino médio, ministrada pelo ex-professor do Campus Nepomuceno, Mateus Coelho;

- Conscientização de Crianças Quanto ao Uso de Energia: direcionada para alunos do ensino fundamental, buscou incentivar a economia de energia e evidenciar a 
importância e significado de Selo Procel, ministrada pelas petianas Letícia Soares Santos e Ignásia Aline Gama Carvalho Ferreira.

Figura 7- Palestra Programa de Educação Tutorial.

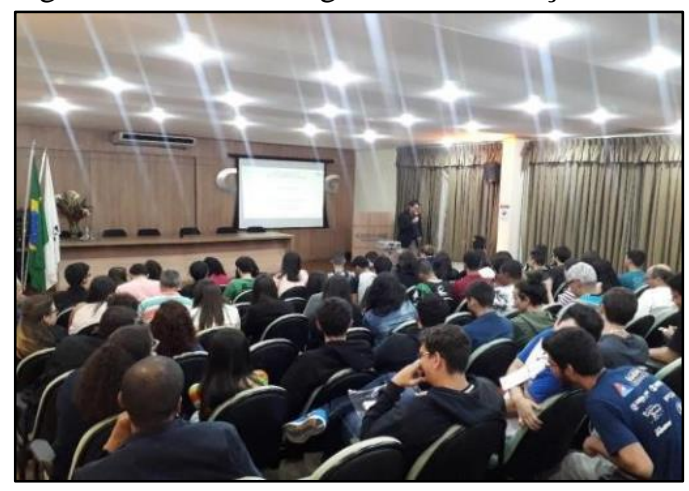

Fonte: $\mathrm{O}$ autor.
Figura 8- Palestra Eficiência Energética.

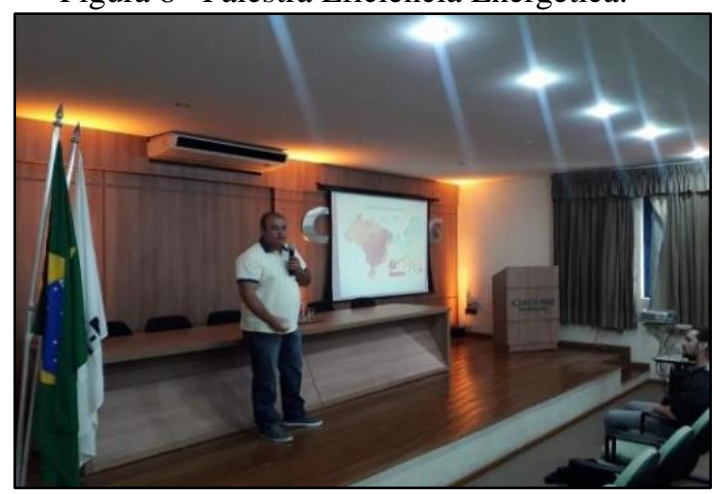

Fonte: $\mathrm{O}$ autor.

Figura 9 - Palestra Conscientização de Crianças Quanto ao Uso de Energia.

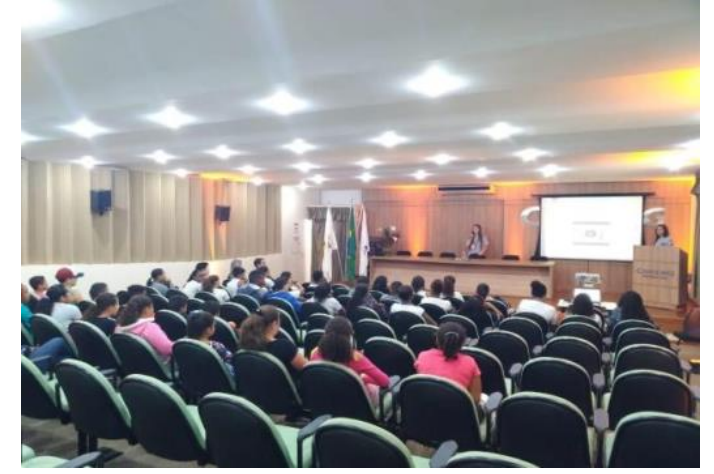

Fonte: $\mathrm{O}$ autor.

\subsection{Mesa redonda e Minicurso}

A mesa redonda com o tema "Desafios acerca do uso consciente da energia no contexto cotidiano da cidade de Nepomuceno" guiada pelos professores: Ítalo Arthur João Wilson Silva Meireles, Márcio Wladimir Santana e Andrei de Oliveira Barra, foi aberta a toda comunidade, e teve como objetivo a realização de um debate com a população a respeito de maneiras eficientes de se utilizar a energia elétrica.

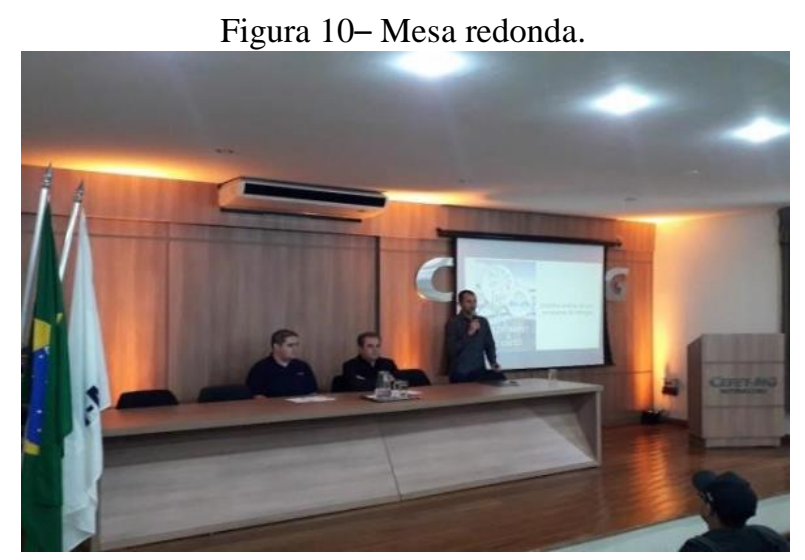

Fonte: $\mathrm{O}$ autor. 
Com o objetivo de apresentar conceitos básicos sobre energia elétrica e os principais cuidados que se deve tomar ao lidar com a mesma, foi ofertado o minicurso "Medidas de Segurança Residencial", realizado pelos petianos Iago Monteiro Vilela e Sara Luiza da Silva voltado para alunos do ensino médio da região.

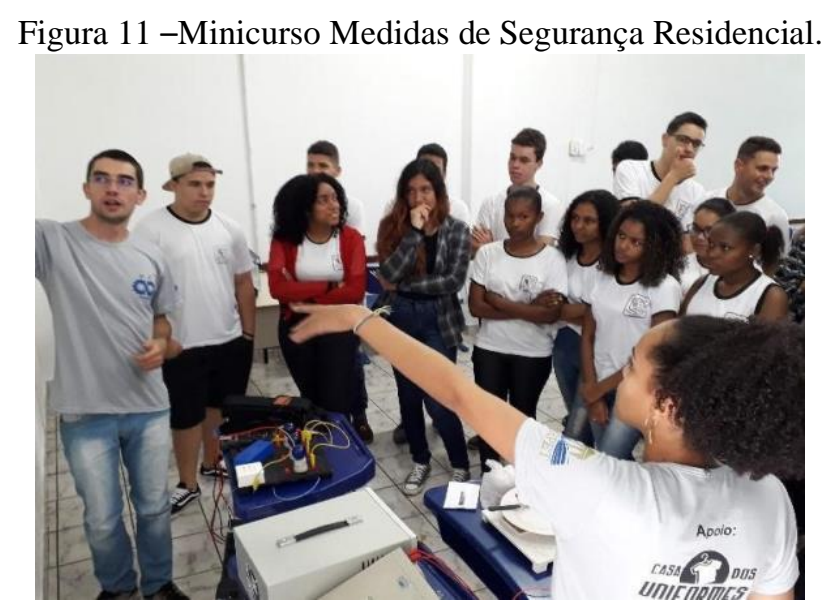

Fonte: $\mathrm{O}$ autor.

\subsection{Apresentação de trabalhos}

Os Grupos PETs da Universidade Federal de Lavras realizaram as apresentações dos trabalhos desenvolvidos pelos grupos ao longo do programa, com o intuito de realizar uma troca de experiências entre os PETs, assim como, enfatizar a importância da tríade ensino, pesquisa e extensão tanto para a comunidade interna quanto para a comunidade externa.

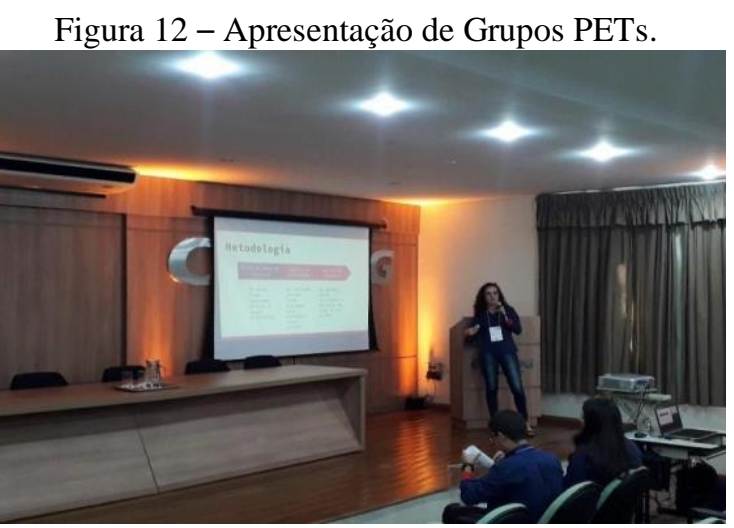

Fonte: $\mathrm{O}$ autor.

\section{CONSIDERAÇÕES FINAIS}

O PETEE CEFET-MG Campus Nepomuceno desenvolve inúmeras oportunidades de atividades como minicursos, palestras e realizações de eventos. Além disso, os petianos através de eventos técnico-científicos têm levado conhecimento à comunidade externa ao Campus, buscando a troca de conhecimentos em conceitos relacionados à tríade: ensino, pesquisa e extensão, visando desenvolver conhecimentos que fortalecerão a relação acadêmica e social.

Os eventos realizados trouxeram vários desafios e aprendizados, por meio das ações executadas. Os petianos aperfeiçoaram e adquiriram novos saberes e habilidades, como o 
trabalho em grupo, análises críticas, e o compromisso com a ética e a cidadania. E devido ao contato com a comunidade externa, o grupo solidificou valores que reforçam a consciência social. A troca de conhecimentos entre diferentes grupos PETs também foi de suma importância pois além da aproximação destes grupos e do início de novas amizades, as experiências vividas por um grupo fomentam novas ideias para outros PETs.

Com a apresentação dos projetos, foi possível observar uma maneira diferente de ensinar e aprender engenharia. Pois, através deles os participantes tiveram que fazer uma reflexão quanto ao seu aprendizado para poderem apresentar seu grupo e os trabalhos já realizados por ele. Tiveram também que aprofundar seus conhecimentos para poderem ministrar mini-cursos e realizar palestras.

O grupo PETEE estimula os discentes em seu desenvolvimento acadêmico, pessoal e profissional, concomitantemente traz consigo oportunidades tanto para seus integrantes, quanto para os alunos da graduação, ensino médio integrado e técnico. Para os participantes do grupo, cada projeto realizado se torna um engrandecimento da formação pessoal e acadêmica, ressaltando em especial o senso de responsabilidade e trabalho em equipe que vem com a organização e execução dessas atividades.

\section{Agradecimentos}

Gostaríamos de agradecer de forma singular à Instituição, em especial a toda equipe de Gestão do CEFET-MG, pelo apoio e incentivo ao evento. Agradecemos, do mesmo modo, a Diretoria de Graduação (DIRGRAD), a fundação CEFET Minas, a Diretoria de Extensão e Desenvolvimento Comunitário (DEDC), a Diretoria de Pesquisa e Pós-Graduação (DPPG), a Empresa Júnior (VRI Jr.) e aos demais patrocinadores por toda assistência e estímulo, sendo estes de suma importância para a realização dos eventos.

\section{REFERÊNCIAS}

BRASIL. Constituição Federal de 1988. Emenda constitucional n ${ }^{\circ} 11$, Art.207 de 30 abril de 1996.

GUIMARÃES, V. A. L.; HAYASHI, M. C. P. I. A comunicação da ciência em eventos científicos na visão de pesquisadores. In Em Questão, Porto Alegre, v.22, n.3, p.161-138, set/dez. 2016.

LIMA, J. E. C. et al. A importância da extensão universitária na formação profissional: Experiência vivenciada por alunos do curso de Farmácia. In II Congresso Brasileiro de Ciências da Saúde (CONBRACIS). 2017.

Ministério da Educação - Secretaria de Educação Superior (SESU). Manual de Orientações Básicas PET. 2002.

NUNES, A.L. P. F.; SILVA, M. B. C. A extensão universitária no ensino superior e a sociedade. In Mal-Estar e Sociedade, v. Ano IV, p. 119-133. 2012.

SANTANA, C. C. et al. A importância da organização de eventos acadêmicos na formação do Biólogo: A iniciativa do Biovertentes. In Extensão, Uberlândia, v.13, n.1, p. 51-60, jan. -jun. 2014. 


\title{
IV PET GROUP MEETING CEFET-MG AND I REGIONAL PET GROUP MEETING: IV INTERPET AND I ERPET
}

\begin{abstract}
This This article is presented as activities developed during the IV Meeting of PET Groups of CEFET-MG (InterPET) and I Regional Meeting of PET groups (ERPET), which takes place simultaneously from 29 to 31 August 2019, on the CEFET campus -MG Nepomuceno. InterPET is an event held since 2016 on the CEFET-MG campus, with the objective of discussing and proposing changes to improve the CEFET-MG PET groups, as well as, relating the activities carried out by each group. ERPET is an event conceived by the PET EngenhariaEngenharia group on the CEFET-MG campus, which aimed to discuss as part of the Tutorial Education Program with PET groups in the region, such as, for example, exporting the studies carried out by each group. In addition to the Petian community, the event was opened in the external community. During the ERPET program, several activities were selected for students from the Municipal and State schools in the city of Nepomuceno. With the realization of events, it was possible to elucidate the importance of high school, research and extension, both for the Petiana community and for the external community, contributing to a reflection on the social functions of PET groups, as transforming agents and within the university.
\end{abstract}

Keywords: PET. Extension. Teaching. Education. 